Research Article

\title{
Digital Device Use, Computer Vision Syndrome, and Sleep Quality among an African Undergraduate Population
}

\author{
Prince Kwaku Akowuah (D), ${ }^{1}$ Augustine N. Nti, ${ }^{2}$ Stephen Ankamah-Lomotey, ${ }^{1}$ \\ Asafo Agyei Frimpong, ${ }^{1}$ Jeremiah Fummey, ${ }^{1}$ Prince Boadi, ${ }^{1}$ Kofi Osei-Poku, ${ }^{1}$ \\ and Joseph Adjei-Anang ${ }^{1}$ \\ ${ }^{1}$ Department of Optometry, Kwame Nkrumah University of Science and Technology, Kumasi, Ghana \\ ${ }^{2}$ Newlands Medical Centre, Koforidua, Ghana \\ Correspondence should be addressed to Prince Kwaku Akowuah; prince.k.akowuah@gmail.com
}

Received 23 November 2020; Revised 7 March 2021; Accepted 10 March 2021; Published 18 March 2021

Academic Editor: Hamidreza Karimi-Sari

Copyright (C) 2021 Prince Kwaku Akowuah et al. This is an open access article distributed under the Creative Commons Attribution License, which permits unrestricted use, distribution, and reproduction in any medium, provided the original work is properly cited.

\begin{abstract}
Background. The purpose of the study was to determine the prevalence of computer vision syndrome (CVS) and poor sleep quality among university students and assess the relationship between digital device usage, CVS, and sleep quality. Methods. A crosssectional study including undergraduate students was conducted in Ghana between January-March 2020. Information on digital device use and CVS symptoms was collected using a structured questionnaire. Sleep quality was assessed using the Pittsburgh Sleep Quality Index (PSQI). Logistic regression was used to determine the relationship between CVS and digital device use behavior, and linear regression analysis was used to explore the association between sleep quality and digital device use behavior. Statistical significance was set at $p<0.05$. Results. Mean (SD) age of participants was 20.95 (1.68) years and most (54.97\%) of them were females. The prevalence of CVS was $64.36 \%$. Factors associated with CVS included hours of digital device use per day $(\mathrm{OR}=4.1, p<0.001)$, years of digital device use $(\mathrm{OR}=3.0, p<0.001)$, adjustment of digital device screen contrast to the surrounding brightness $(\mathrm{OR}=1.95, p=0.014)$, and presence of glare $(\mathrm{OR}=1.79, p=0.048)$. Prevalence of poor sleep quality was $62.43 \%$. There was a significant association between poor sleep quality and number of years participants had used a digital device $(p=0.015)$ and the number of hours they used a digital device per day $(p=0.005)$. Conclusion. There is a high prevalence of both CVS and poor sleep quality among undergraduate students in Ghana. This represents a significant public health issue that needs attention.
\end{abstract}

\section{Introduction}

Computer vision syndrome (CVS) is a cluster of eye and vision problems experienced in relation to, or during, the use of digital devices. Symptoms of CVS include but are not limited to headaches, blurred vision, double vision, eye strain and fatigue, teary eyes, sensitivity to light, burning sensation, and ocular surface dryness [1]. The public health significance of CVS cannot be overstated and demands the attention of researchers, clinicians, and policy makers. Computer vision syndrome is the leading occupational hazard of the twenty-first century, and its symptoms affect $63 \%-89 \%$ of computer users depending on the population studied [2-6]. Approximately 60 million computer users globally suffer from CVS, with nearly one million new cases diagnosed each year $[7,8]$. Computer vision syndrome reduces work efficiency and productivity, causing increased work errors and reduced job satisfaction [9]. Symptoms of CVS can have ocular etiologies such as uncorrected refractive errors, accommodative dysfunction, and binocular vision abnormalities [10] or ergonomic etiologies such as poor sitting position, improper viewing distance and angle, poor display resolution and contrast, poor lighting, and imbalance between display and environmental lighting [11-13].

The effect of prolonged digital device use on sleep has now become a concern [14]. Prolonged digital device use results in significant reduction in sleep amount and quality 
$[15,16]$. Inadequate sleep is associated with poor work and school performance, reduced productivity, lack of energy, higher risk of weight gain, and depression [17]. One important factor influencing sleep quality in the twenty-first century is the use of digital devices $[15,16]$. Digital device use, especially at night, has been shown to impair sleep and reduce sleep quality [18]. Use of digital devices disrupts the circadian rhythm by suppressing the release of melatonin, a sleep-inducing hormone. While light of any kind or white light in general can suppress melatonin secretion, this is principally attributed to the amount of blue light emitted by digital devices [15].

University students constitute a population with massive use of digital devices especially computers and smartphones. Although over $80 \%$ of university students in Ghana own and use a laptop or desktop computer $[19,20]$, there is no available data on CVS among university students in Ghana and Africa at large. Moreover, sleep quality is expected to be poorer in students with heavy digital device use [21]. Poorer sleep quality will be especially detrimental to university students as sleep plays an essential role in memorization, concentration, and learning processes [22]. No attempt has yet been made to examine the relationship between digital device use and sleep quality among undergraduates in Africa. The aim of the current study was to examine CVS and its association with sleep quality among an undergraduate population in Ghana.

\section{Methods}

This study was a cross-sectional study conducted in Kwame Nkrumah University of Science and Technology (KNUST), Ghana, between January-March 2020. Kwame Nkrumah University of Science and Technology is a public university located in Kumasi, in the Ashanti region.

2.1. Sample Size and Sampling. Sample size was calculated using the formula

$$
\begin{aligned}
& n=\frac{N * X}{(X+N-1)}, \\
& X=\frac{Z_{\alpha / 2}^{2} * p *(1-p)}{d},
\end{aligned}
$$

where $n$ represents the sample size, $N$ is the population size, $Z_{\alpha / 2}$ is the critical value at $\alpha / 2, d$ is the margin of error, and $p$ is the sample proportion. The prevalence of CVS reported by Dessie et al. [23] (69.5\%) was chosen as the sample of proportion. Assuming a design effect of 1.5, the calculated sample size was 326 . Allowing for $20 \%$ spoilt data, the final sample size was 391 .

There are six halls of residence in KNUST (University Hall, Unity Hall, Africa Hall, Republic Hall, Independence Hall, and Queens Hall). For each hall of residence, 33 rooms were randomly selected by balloting. Simple random sampling was then used to select two of four occupants of each room for participation in the study. Sixtyfive (65) participants were recruited from all halls of residence, except the Unity Hall, where 66 participants were recruited. All participants were students of KNUST. All questionnaires were self-administered by participants. Participants of all ages were included. Only individuals who use electronic/digital devices were included in the study. Individuals with any diagnosed ocular disease and coffee takers (consuming coffee $\geq 3$ days/week) were excluded from the study.

2.2. Questionnaires. Participants' sociodemographic data and symptoms of CVS and associated factors were obtained using a modified form of the study-specific questionnaire used in the study by Aseefa et al. [4]. Diagnosis of CVS was defined as the presence of two or more symptoms experienced during or after prolonged digital device use over the immediate past month. For this study, digital device use was defined as laptop, desktop, and tablet use. Every item on the questionnaire was explained to participants by members of the research team. The Pittsburgh Sleep Quality Index (PSQI) is a self-administered questionnaire that assesses the quality of sleep over a one-month period. The PSQI is a validated and reliable questionnaire for assessing sleep quality in both research and clinical settings [24]. The PSQI assesses seven components of sleep. The PSQI components are subjective sleep quality, sleep latency, sleep duration, habitual sleep efficiency, sleep disturbance, use of sleep medication, and daytime dysfunction. All components are scored on a $0-3$ scale. A global PSQI score (in the range 0-21) is obtained from the sum of the seven components. Lower global PSQI score indicates good/healthier sleep quality and high PSQI indicates poor sleep quality [24]. For this study, global PSQI score $\leq 5$ was classified as "good sleep quality," and global PSQI score $>5$ was classified as "poor sleep quality" [24].

2.3. Ethical Consideration. Written informed consents were obtained from all participants prior to their participation in the study. All study protocols complied with the tenets of the Declaration of Helsinki. Approval for the study was obtained from the Institutional Review Committee of Kwame Nkrumah University of Science and Technology.

2.4. Statistical Analysis. Data was analyzed with Statistical Package for Social Sciences (SPSS) software version 25.0. Mean, standard deviation, and percentages were used to describe data, where appropriate. Binary logistic regression analysis was performed to determine the relationship between CVS and participant characteristics and digital device use behavior, and linear regression analysis was used to explore the association between the global PSQI, its individual components and years of digital device use, and digital device use per day (hours). Chi-square test was conducted to examine association between CVS and sleep quality. At $95 \%$ confidence interval, $p \leq 0.05$ was considered statistically significant. 


\section{Results}

A total of 391 individuals participated, with 362 valid data obtained. Table 1 provides a summary of participants' characteristics. The majority (54.97\%) of the participants were females. The mean (SD) age of participants was 20.95 (1.68) years.

3.1. Digital Device Use Behavior. Table 2 provides a summary of the digital device use behavior of study participants. The majority $(60.22 \%)$ of participants had been using digital device for $\geq 5$ years. Approximately three-fourth (72.38\%) spent three hours or more on the digital device per day.

3.2. Computer Vision Syndrome. The prevalence of CVS was $64.36 \%$ (95\% CI: $59.2 \%-69.3 \%)$. Table 3 provides a summary of the frequency of CVS according to participant characteristics and digital device use behavior. The most common symptoms reported were teary eyes (40.06\%), eye fatigue (35.36\%), and eye strain (34.81\%). A summary of the symptoms reported by participants is presented in Figure 1.

After adjusting for the effects of age, gender, and habitual bedtime smartphone use, multiple binary logistic regression revealed that the following factors were significantly associated with CVS: years of digital device use $(\mathrm{OR}=3.0,95 \% \mathrm{CI}: 2.12-4.01, p<0.001)$, digital device use per day (hours) $(\mathrm{OR}=4.1,95 \% \mathrm{CI}$ : 3.16-6.27, $p<0.001)$, frequent breaks during digital device use $(\mathrm{OR}=0.51,95 \%$ CI: $0.27-0.81, p=0.02$ ), adjustment of digital device screen contrast to surrounding brightness ( $\mathrm{OR}=1.9595 \%$ CI: $1.37-5.12, p=0.02)$, glare experience $(\mathrm{OR}=1.79,95 \%$ CI: $1.21-3.17, p=0.04)$, and spectacle wear $(\mathrm{OR}=0.46$, 95\% CI: $0.36-0.92, p=0.01$ ).

3.3. Sleep Quality and Digital Device Use. The overall mean (SD) global PSQI was 6.49 (2.75). The mean (SD) score for subjective sleep quality was 0.92 (0.69); sleep latency, 1.65 (1.12); sleep duration, 1.57 (0.67); habitual sleep deficiency, $0.63(0.45)$; sleep disturbance, $1.04(0.4)$; use of sleep medication, 0.12(0.03); daytime dysfunction, 0.58 (0.29). Table 4 gives a summary of the global PSQI and score on the individual component with years of digital device use and duration of digital device use per day. The prevalence of poor sleep quality among participants was $62.43 \%$ (95\% CI: 57.2\%-67.4\%). After controlling for age, gender, BMI, and habitual bedtime smartphone, multiple binary logistic regression analysis revealed that "years of digital device use" was associated with poor overall sleep quality $(p=0.02)$, poor subjective sleep quality $(p=0.001)$, higher sleep latency $(p=0.01)$, sleep disturbance $(p=0.004)$, and daytime dysfunction $(p=0.001)$. Digital device use per day was also associated with poor overall sleep quality $(p=0.005)$, poor subjective sleep quality $(p=0.001)$, higher sleep latency $(p=0.003)$, shorter sleep duration $(p=0.02)$, and habitual sleep efficiency $(p=0.005)$.
TABLE 1: Characteristics of study participants.

\begin{tabular}{lcc}
\hline Characteristics & & Frequency (\%) \\
\hline \multirow{2}{*}{ Gender } & Male & $163(45.03)$ \\
& Female & $199(54.97)$ \\
\hline \multirow{3}{*}{ Age (years) } & $\leq 20$ & $147(40.61)$ \\
& $21-25$ & $206(56.91)$ \\
\multirow{3}{*}{ BMI $\left(\mathrm{kg} / \mathrm{m}^{2}\right)$} & $26-30$ & $9(2.48)$ \\
\hline \multirow{2}{*}{ Bedtime smartphone use } & $18.5-24.9$ & $268(74.03)$ \\
& $25.0-29.9$ & $79(21.82)$ \\
& $\geq 30.0$ & $15(4.14)$ \\
\hline \multirow{3}{*}{ Year of college } & Yes & $279(77.07)$ \\
& No & $83(22.93)$ \\
\hline \multirow{2}{*}{ Spectacle use } & $1^{\text {st }}$ & $31(8.56)$ \\
& $2^{\text {nd }}$ & $92(25.41)$ \\
\multirow{2}{*}{ Purpose of spectacle wear } & $3^{\text {rd }}$ & $86(23.76)$ \\
& $4^{\text {th }}$ & $153(42.27)$ \\
\hline & Yes & $177(48.90)$ \\
& Digital device use & $37(20.90)$ \\
& Other purpose & $18(10.17)$ \\
\hline
\end{tabular}

3.4. Computer Vision Syndrome and Sleep Quality. The mean (SD) PSQI score among participants with CVS was 7.09 (2.81) and without CVS was 5.89 (2.67). The prevalence of poor sleep quality among participant with CVS was $67.24 \%$ and without CVS was $54.62 \%$. The chi-square test revealed a significant association between CVS and sleep quality (Pearson's chi-square $=5.68, p=0.02$ ).

\section{Discussion}

To the best of the authors' knowledge, the current study is the first study assessing digital device use, computer vision syndrome, and sleep quality among any African population. The prevalence of CVS in the current study was $64.36 \%$. Other studies have reported prevalence of CVS ranging from $63 \%$ to $89 \%$ in various populations [3-6]. Eye fatigue and eye strain were the common CVS symptoms reported in the study. This is consistent with reports from similar studies [4-6]. Prolonged accommodation and convergence on close objects such as a digital device screen put strain on the visual system leading to visual discomfort including eye fatigue and eye strain [25]. Additionally, frequently changing focus from digital devices to printed material or changing focus from one digital device to another can put strain on the visual system, leading to reports of eye fatigue and eye strain $[25,26]$.

Consistent with reports from similar studies in Ethiopia [4] and India [27], there was a significant association between taking less frequent breaks from digital device use and CVS symptoms. Working on digital devices without taking breaks forces the eye to maintain focus for long leading to visual fatigue and symptoms of asthenopia [28]. This result is significant to both clinicians and students because, with the current COVID-19 pandemic, several universities moved their lectures online and digital device usage among university students worldwide is expected to increase. With almost all 
TABLE 2: Digital device use behavior of study participants.

\begin{tabular}{|c|c|c|}
\hline Digital device use behavior & & Frequency $(\%)$ \\
\hline \multirow{3}{*}{ Years of digital device use } & $<5$ & $144(39.78)$ \\
\hline & $5-10$ & $91(25.14)$ \\
\hline & $>10$ & $127(35.08)$ \\
\hline \multirow{3}{*}{ Duration of digital device use per day (hours) } & $<3$ & $100(27.62)$ \\
\hline & $3-6$ & $152(41.99)$ \\
\hline & $>6$ & $110(30.39)$ \\
\hline \multirow{2}{*}{ Breaks during digital device use } & Yes & $275(75.97)$ \\
\hline & No & $87(24.03)$ \\
\hline \multirow{2}{*}{ Length of break (minutes) } & $<20$ & $148(53.82)$ \\
\hline & $>20$ & $127(46.18)$ \\
\hline \multirow{2}{*}{ Voluntary blinks during digital device use } & Yes & $201(55.52)$ \\
\hline & No & $161(44.48)$ \\
\hline \multirow{2}{*}{ Digital device brightness/contrast adjustment } & Yes & $296(81.77)$ \\
\hline & No & $66(18.23)$ \\
\hline \multirow{2}{*}{ Glare experience } & Yes & $126(34.81)$ \\
\hline & No & $236(65.19)$ \\
\hline \multirow{2}{*}{ Antiglare device use } & Yes & $50(13.81)$ \\
\hline & No & $312(86.19)$ \\
\hline
\end{tabular}

TABle 3: CVS by participant characteristics and digital device use behavior.

\begin{tabular}{|c|c|c|}
\hline Participants characteristic & & CVS frequency (\%) \\
\hline \multirow{2}{*}{ Gender } & Male & $102(62.58)$ \\
\hline & Female & $131(65.83)$ \\
\hline \multirow{3}{*}{ Age } & $\leq 20$ & $95(64.63)$ \\
\hline & $21-25$ & $134(65.05)$ \\
\hline & $26-30$ & $4(44.44)$ \\
\hline \multirow{4}{*}{ Year of college } & $1^{\text {st }}$ & $17(54.84)$ \\
\hline & $2^{\text {nd }}$ & $61(66.3)$ \\
\hline & $3^{\text {rd }}$ & $59(68.6)$ \\
\hline & $4^{\text {th }}$ & $96(62.75)$ \\
\hline \multirow{3}{*}{ Years of digital device use } & $<5$ & $50(36.23)$ \\
\hline & $5-10$ & $76(80)$ \\
\hline & $>10$ & $107(82.95)$ \\
\hline \multirow{3}{*}{ Digital device use per day (hours) } & $<3$ & $37(36.64)$ \\
\hline & $3-6$ & $106(70.2)$ \\
\hline & $>6$ & $90(81.82)$ \\
\hline \multirow{2}{*}{ Frequent break during digital device use } & Yes & $168(61.09)$ \\
\hline & No & $65(74.71)$ \\
\hline \multirow{2}{*}{ Digital device brightness/contrast adjustment } & Yes & $188(63.51)$ \\
\hline & No & $45(66.18)$ \\
\hline \multirow{2}{*}{ Anti-glare device use } & Yes & $34(68.0)$ \\
\hline & No & $199(63.78)$ \\
\hline
\end{tabular}

students taking classes online, the average amount of time spent on digital devices is expected to increase. This has the potential to cause an increase in the prevalence and severity of CVS in these populations. For some of these people, symptoms of CVS can lead to reductions in quality of life and work productivity [9]. Additionally, this study found that participants who adjusted screen brightness to suit the surrounding lighting conditions had higher odds of reporting symptoms of CVS. While this initially seems counterintuitive, it is important to note that the need to adjust digital device screens to the lighting conditions implies that the lighting condition is inadequate. While adjustments to the digital device screen are helpful, it does not fully eliminate the problems associated with inadequate lighting [7]. Lighting conditions can cause visual discomfort and symptoms of CVS through uneven illumination, reflections off the digital device screen, and discomfort glare. [1] Glare and digital device screen reflection can also lead to reduction in the contrast of the digital device display, degrade the image seen on the screen, and exacerbate visual discomfort. In the current study, wearing spectacles (glasses) was associated with lower odds of developing CVS symptoms. Properly prescribed spectacles can help reduce eye 


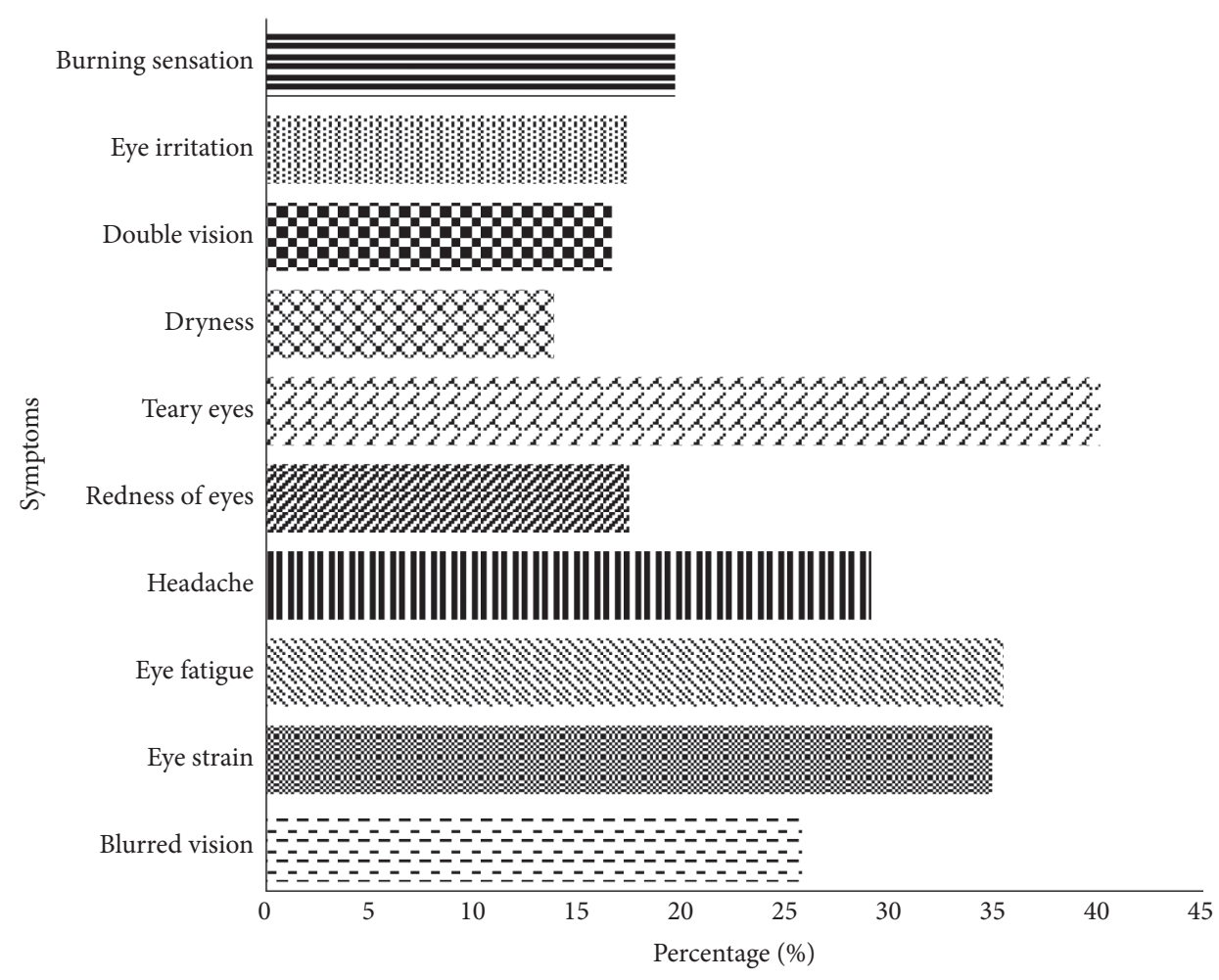

FIGURE 1: Summary of CVS symptoms reported by study participants.

TABLE 4: PSQI total and individual component score with years of digital device use and duration of digital device use per day.

\begin{tabular}{|c|c|c|c|c|c|c|c|c|}
\hline & \multicolumn{4}{|c|}{ Years of digital device use } & \multicolumn{4}{|c|}{ Digital device use per day (hours) } \\
\hline & $\begin{array}{c}<5 \\
\text { Mean }(\mathrm{SD})\end{array}$ & $\begin{array}{c}5-10 \\
\text { Mean (SD) }\end{array}$ & $\begin{array}{c}>10 \\
\text { Mean }(\mathrm{SD})\end{array}$ & $p$ value & $\begin{array}{c}<3 \\
\text { Mean (SD) }\end{array}$ & $\begin{array}{c}3-6 \\
\text { Mean (SD) }\end{array}$ & $\begin{array}{c}>6 \\
\text { Mean (SD) }\end{array}$ & $p$ value \\
\hline Global PSQI & $5.63(2.42)$ & $6.59(2.35)$ & $7.40(3.07)$ & $<0.001$ & $5.59(2.29)$ & $6.26(2.35)$ & $7.63(3.24)$ & $<0.001$ \\
\hline Subjective sleep quality & $0.8(0.62)$ & $0.84(0.62)$ & $1.13(0.78)$ & 0.001 & $0.77(0.53)$ & 0.87 & 1.14 & 0.256 \\
\hline Sleep latency & $1.40(1.20)$ & $1.64(1.21)$ & $1.94(1.59)$ & 0.01 & $1.4(1.1)$ & $1.50(1.19)$ & $2.07(1.58)$ & 0.003 \\
\hline Sleep duration & $1.48(0.83)$ & $1.59(0.69)$ & $1.65(0.75)$ & 0.261 & $1.42(0.85)$ & $1.57(0.77)$ & $1.70(0.67)$ & 0.02 \\
\hline Habitual sleep defic & $0.56(0.61)$ & $0.63(0.60)$ & $0.71(0.89)$ & 0.811 & $0.47(0.34)$ & $0.58(0.47)$ & $0.85(0.73)$ & 0.005 \\
\hline Sleep disturbance & $0.94(0.53)$ & $1.05(0.50)$ & $1.13(0.44)$ & 0.004 & $1.00(0.55)$ & $1.03(0.46)$ & $1.08(0.49)$ & 0.638 \\
\hline Use of sleep medication & $0.11(0.34)$ & $0.15(0.22)$ & $0.1(0.26)$ & 0.893 & $0.09(0.08)$ & $0.11(0.09)$ & $0.15(0.09)$ & 0.613 \\
\hline Daytime dysfunction & $0.35(0.45)$ & $0.69(0.62)$ & $0.76(0.55)$ & 0.001 & $0.46(0.34)$ & $0.62(0.50)$ & $0.65(0.54)$ & 0.825 \\
\hline
\end{tabular}

strain and visual fatigue, especially on digital devices $[29,30]$. Contrary to the finding in the current study, a similar study [4] reported increased odds of CVS development in spectacle wearers. The general explanation given by these studies was that participants might not have been wearing correct prescriptions.

The impact of digital device use on sleep quality is increasingly becoming a concern. With increasing technological advancement and shift to a digital world, digital devices have become a necessary and integral part of our day-to-day life. The majority $(62.43 \%)$ of the participant in the current study had poor sleep quality. This is similar to results from other studies reporting negative effects of excessive digital device use on sleep quality in college students and other populations $[15,18]$. Exposure to digital device screen light can disturb an individual's circadian rhythm [31]. A study in Australia [32] also reported strong associations between high amount of digital device use and general sleep problems. Although a significant association between digital device use and sleep quality was observed in the current study, it is important to acknowledge that this does not prove that digital device use is the direct cause of the poor sleep quality. Other factors such as daily stress [33] and poor sleeping conditions [34] could have possibly contributed to the poor sleep quality observed in this study. The current study also revealed a significant association between sleep latency and both years of digital device use and digital device use per day (hours). Participants with longer years of digital device use and longer hours of digital device use in a day tend to have prolonged sleep latency. A known explanation for the prolonged sleep latency with digital device and other digital device use is the emission of blue light from these devices. Blue light is known to suppress the release of melatonin, a sleep-inducing hormone from the pineal gland, leading to the prolonged sleep latency [31]. 
Another significant finding in the current study was the reduction in sleep duration with increased hours of digital device use per day. There was also a reduction in sleep duration with increasing years of digital device use. Different studies have reported the effects of excessive screen time, internet addiction, and bedtime digital devices use on sleep duration. A study in Taiwan [18] evaluating the relationship between digital device use (internet addiction) and sleep quality in college students reported decreasing sleep duration with increasing internet addiction, thus increasing digital device use and screen time. Habitual sleep efficiency was also significantly associated with digital device use per day (hours). Increased hours of digital device use per day were associated with decreased habitual sleep efficiency. Similar association between digital device use and sleep efficiency was reported by Fobian et al. [35] in their study on the impact of digital media use on adolescent sleep efficiency. It is not surprising that increased hours of digital device use decrease habitual sleep efficiency. Considering the significant reduction in sleep duration and prolonged sleep latency, it was expected that increased digital device use per day would affect the efficiency of habitual sleep.

There also was a significant relationship between sleep disturbance and years of digital device use. The PSQI sleep disturbance component measures sleep interference such as waking up in the middle of the night, feeling too cold or hot, coughing, troubled breathing, and having bad dreams with a high score indicating discomfort or physical impairment of sleep. It is important to acknowledge that these disturbances to sleep might not be a direct effect of digital device use or screen time. However, the effect that digital device use has on the body's physiology could result in physical discomfort. These physical discomforts and their resultant sleep disturbance are of concern as they can affect academic and work performance. Furthermore, there was a significant association between years of digital device use and daytime dysfunction. Although not significant, increasing digital device use per day was associated with increasing daytime dysfunction. A study in Singapore [36] reported a significant association between digital device use in the form of social media (at both daytime and nighttime) and daytime dysfunction (daytime sleepiness), where an increase in social media use was associated with an increase in daytime sleepiness.

A limitation of the current study is that the time of day participants actively use digital devices was not assessed. Assessing the time of the day participants mostly use digital devices would provide an opportunity to analyze if the effects of digital device use on sleep quality depend on the time (day vs. night) of active digital device use, as almost all studies on the topic have concentrated on digital device use at bedtime. This, however, does not significantly impact the strength of the study as both daytime and nighttime use of digital devices have shown to impact the overall sleep quality, sleep latency, and sleep duration [37]. Moreover, the study did not assess if the spectacles prescriptions used by participants were up-todate and if that had any effect on experiencing CVS.
Furthermore, this study employed questionnaires which required participants to self-report information about their digital device use and sleep. This might have been subject to recall bias.

In future studies, there would be a need to assess if spectacles worn by participants contained their optimal refractive corrections or had features adapted for digital device use such as antireflective coatings and how it affects their experience of CVS.

\section{Conclusion}

In conclusion, there is a high prevalence of both CVS and poor sleep quality among undergraduate students in Ghana. This represents a significant public health issue that needs attention.

\section{Data Availability}

The data can be obtained from the corresponding author upon request.

\section{Conflicts of Interest}

The authors have no conflicts of interest to declare.

\section{References}

[1] C. Blehm, S. Vishnu, A. Khattak, S. Mitra, and R. W. Yee, "Computer vision syndrome: a review," Survey of Ophthalmology, vol. 50, no. 3, pp. 253-262, 2005.

[2] N. A. Charpe and V. Kaushik, "Computer vision syndrome (CVS): recognition and control in software professionals," Journal of Human Ecology, vol. 28, no. 1, pp. 67-69, 2009.

[3] R. Akinbinu, "Knowledge of computer vision syndrome among computer users in the workplace in Abuja, Nigeria," Journal of Physiology and Pathophysiology, vol. 4, no. 4, pp. 58-63, 2013.

[4] N. L. Assefa, D. Z. Weldemichael, H. W. Alemu, and D. H. Anbesse, "Prevalence and associated factors of computer vision syndrome among bank workers in Gondar city, Northwest Ethiopia," Clinical Opthomology, vol. 9, pp. 67-76, 2015.

[5] P. Ranasinghe, W. S. Wathurapatha, Y. S. Perera et al., "Computer vision syndrome among computer office workers in a developing country: an evaluation of prevalence and risk factors," BMC Research Notes, vol. 9, 2016.

[6] S. C. Reddy, C. Low, Y. Lim, L. Low, F. Mardina, and M. Nursaleha, "Computer vision syndrome: a study of knowledge and practices in university students," Nepalese Journal of Ophthalmology, vol. 5, no. 2, pp. 161-168, 2013.

[7] S. Gowrisankaran and J. E. Sheedy, "Computer vision syndrome: a review," Work, vol. 11, pp. 25-29, 2015.

[8] S. A. Randolph, "Computer vision syndrome," Workplace Health \& Safety, vol. 65, no. 7, 2017.

[9] J. R. Hayes, J. E. Sheedy, J. A. Stelmack, and C. A. Heaney, "Computer use, symptoms, and quality of life," Opthomology Visual Science, vol. 84, pp. 738-744, 2007.

[10] M. Rosenfield, "Computer vision syndrome: a review of ocular causes and potential treatments," Ophthalmic and Physiological Optics, vol. 31, no. 5, pp. 502-515, 2011.

[11] S. B. Boadi-Kusi, S. L. Abu, G. O. Acheampong, P. O. W. Adueming, and E. K. Abu, "Association between 
poor ergophthalmologic practices and computer vision syndrome among university administrative staff in Ghana," Journal of Environment Public Health, vol. 2020, 2020.

[12] M. Sánchez-Brau, B. Domenech-Amigot, F. Brocal-Fernández, J. A. Quesada-Rico, and M. Seguí-Crespo, "Prevalence of computer vision syndrome and its relationship with ergonomic and individual factors in presbyopic VDT workers using progressive addition lenses," International Journal of Environmental Research and Public Health, vol. 17, no. 3, 2020.

[13] J. R. Anshel, "Visual ergonomics in the workplace," $A A O H N$ Journal, vol. 55, no. 10, pp. 414-420, 2007.

[14] X. Mei, Q. Zhou, X. Li, P. Jing, X. Wang, and Z. Hu, "Sleep problems in excessive technology use among adolescent: a systemic review and meta-analysis," Sleep Science Practise, vol. 2, 2018.

[15] C. Fuller, E. Lehman, S. Hicks, and M. B. Novick, "Bedtime use of technology and associated sleep problems in children," Global Pediatric Health, vol. 4, 2017.

[16] S. Thomée, A. Härenstam, and M. Hagberg, "Mobile phone use and stress, sleep disturbances, and symptoms of depression among young adults - a prospective cohort study," BMC Public Health, vol. 12, 2011.

[17] A. Smaldone, J. C. Honig, and M. W. Byrne, "Sleepless in America: inadequate sleep and relationships to health and well-being of our nation's children," Pediatrics, vol. 119, no. 1, pp. S29-S37, 2007.

[18] P. H. Lin, Y. C. Lee, K. L. Chen, P. L. Hsieh, S. Y. Yang, and Y. L. Lin, "The relationship between sleep quality and internet addiction among female college students," Frontiers in Neuroscience, vol. 13, 2019.

[19] S. Dery, F. D. Vroom, A. Godi, S. Afagbedzi, and D. Dwomoh, "Knowledge and use of information and communication technology by health sciences students of the University of Ghana," Ghana Medical Journal, vol. 50, no. 3, pp. 180-188, 2016.

[20] S. Farouq and F. Mensah, "Ghanaian tertiary students' use of ICT," Global Journal of Human-Social Science, vol. 19, pp. 13-18, 2017.

[21] A. Mohammadbeigi, R. Absari, F. Valizadeh et al., "Sleep quality in medical students; the impact of over-use of mobile cell-phone and social networks," Journal of Research Health Science, vol. 19, pp. 659-671, 2016.

[22] N. Hennies, M. A. Lambon Ralph, M. Kempkes, J. N. Cousins, and P. A. Lewis, "Sleep spindle density predicts the effect of prior knowledge on memory consolidation," The Journal of Neuroscience, vol. 36, no. 13, pp. 3799-3810, 2016.

[23] A. Dessie, F. Adane, A. Nega, S. D. Wami, and D. H. Chercos, "Computer vision syndrome and associated factors among computer users in debre tabor town, northwest Ethiopia," Journal of Environmental Public Health, vol. 2018, 2018.

[24] Q.-Y. Zhong, B. Gelaye, S. E. Sánchez, and M. A. Williams, "Psychometric properties of the Pittsburgh sleep quality index (PSQI) in a cohort of Peruvian pregnant women," Journal of Clinical Sleep Medicine, vol. 11, no. 8, pp. 869-877, 2015.

[25] A. L. Sheppard and J. S. Wolffsohn, "Digital eye strain: prevalence, measurement and amelioration," BMJ Open Ophthalmology, vol. 3, Article ID e000146, 2018.

[26] J. E. Sheedy and S. D. Parsons, "The video display terminal eye clinic: clinical report," Optometry and Vision Science, vol. 67, no. 8, pp. 622-626, 1990.

[27] S. Agarwal, D. Goel, and A. Sharma, "Evaluation of the factors which contribute to the ocular complaints in computer users,"
Journal of Clinical Diagnostic Research, vol. 7, pp. 331-335, 2013.

[28] D. Bhanderi, S. Choudhary, and V. Doshi, "A communitybased study of asthenopia in computer operators," Indian Journal of Ophthalmology, vol. 56, no. 1, pp. 51-55, 2008.

[29] S. P. Butzon, J. E. Sheedy, and E. Nilsen, "The efficacy of computer glasses in reduction of computer worker symptoms," Optometry (St. Louis, MO, USA), vol. 73, no. 4, pp. 221-230, 2002.

[30] G. Horgen, A. Aarås, and M. Thoresen, "Will visual discomfort among Visual Display Unit (VDU) users change in development when moving from single vision lenses to specially designed VDU progressive lenses?" Optometry and Vision Science, vol. 81, no. 5, pp. 341-349, 2004.

[31] C. Moderie, S. Van der Maren, and M. Dumont, "Circadian phase, dynamics of subjective sleepiness and sensitivity to blue light in young adults complaining of a delayed sleep schedule," Sleep Medicine, vol. 34, pp. 148-155, 2017.

[32] A. L. Gamble, A. L. D'Rozario, D. J. Bartlett et al., "Adolescent sleep patterns and night-time technology use: results of the Australian broadcasting corporation's big sleep survey," PLoS ONE, vol. 9, Article ID e111700, 2014.

[33] J. M. Blaxton, C. S. Bergeman, B. R. Whitehead, M. E. Braun, and J. D. Payne, "Relationships among nightly sleep quality, daily stress, and daily affect," Journals of Gerontology Series B: Psychological Sciences and Social Sciences, vol. 72, pp. 363372, 2017.

[34] I. Altun, N. Cinar, and C. Dede, "The contributing factors to poor sleep experiences in according to the university students: a cross-sectional study," Journal of Research in Medical Sciences: The Official Journal of Isfahan University of Medical Sciences, vol. 17, no. 6, pp. 557-561, 2012.

[35] A. D. Fobian, K. Avis, and D. C. Schwebel, "Impact of media use on adolescent sleep efficiency," Journal of Developmental \& Behavioral Pediatrics, vol. 37, no. 1, pp. 9-14, 2016.

[36] A. M. A. Nasirudeen, L. Lee Chin Adeline, K. Wat Neo Josephine, L. Lay Seng, and L. Wenjie, "Impact of social media usage on daytime sleepiness: a study in a sample of tertiary students in Singapore," Digital Health, vol. 3, 2017.

[37] M. Hysing, S. Pallesen, K. M. Stormark, R. Jakobsen, A. J. Lundervold, and B. Sivertsen, "Sleep and use of electronic devices in adolescence: results from a large population-based study," BMJ Open, vol. 5, Article ID e006748, 2015. 